\title{
A polymerase chain reaction-based methodology to detect gene doping
}

\author{
Adam Carter • Martin Flueck
}

Received: 23 April 2011 / Accepted: 29 July 2011 / Published online: 17 August 2011

(C) Springer-Verlag 2011

\begin{abstract}
The non-therapeutic use of genes to enhance athletic performance (gene doping) is a novel threat to the World of Sports. Skeletal muscle is a prime target of gene therapy and we asked whether we can develop a test system to produce and detect gene doping. Towards this end, we introduced a plasmid (pCMV-FAK, $3.8 \mathrm{~kb}, 50 \mu \mathrm{g}$ ) for constitutive expression of the chicken homologue for the regulator of muscle growth, focal adhesion kinase (FAK), via gene electro transfer in the anti-gravitational muscle, $m$. soleus, or gastrocnemius medialis of rats. Activation of hypertrophy signalling was monitored by assessing the ribosomal kinase p70S6K and muscle fibre cross section. Detectability of the introduced plasmid was monitored with polymerase chain reaction in deoxyribonucleic acids (DNA) from transfected muscle and serum. Muscle transfection with pCMV-FAK elevated FAK expression 7- and 73-fold, respectively, and increased mean cross section by
\end{abstract}

Communicated by Håkan Westerblad.

Electronic supplementary material The online version of this article (doi:10.1007/s00421-011-2113-y) contains supplementary material, which is available to authorized users.

A. Carter · M. Flueck $(\bowtie)$

School of Healthcare Science, Institute for Biomedical Research into Human Movement and Health, University of Manchester, Manchester M1 5GD, UK

e-mail:m.flueck@mmu.ac.uk

M. Flueck

Centre for Cardiovascular Sciences,

University of Birmingham Medical School,

Birmingham B15 2TT, UK

M. Flueck

Department of Cardiovascular Surgery,

Insel-Hospital, Berne, 3010 Berne, Switzerland
52 and $16 \%$ in targeted muscle fibres of soleus and gastrocnemius muscle 7 days after gene electro transfer. Concomitantly p70S6K content was increased in transfected soleus muscle $(+110 \%)$. Detection of the exogenous plasmid sequence was possible in DNA and cDNA of muscle until 7 days after transfection, but not in serum except close to the site of plasmid deposition, $1 \mathrm{~h}$ after injection and surgery. The findings suggest that the reliable detection of gene doping in the immoral athlete is not possible unless a change in the current practice of tissue sampling is applied involving the collection of muscle biopsy close to the site of gene injection.

Keywords Sports - Athlete - Doping · Hypertrophy · Gene $\cdot$ Therapy $\cdot$ FAK $\cdot$ PCR $\cdot$ Threshold

\section{Introduction}

Gene doping is a new threat to the world of sports which arose as a spin off of gene therapy (Andersen et al. 2000; Filipp 2007; Sweeney 2004). It is based on the introduction of genetic vectors into cells (transfection) which carry the message for the expression of transcripts and proteins. Today, this is feasible for a number of cell types, i.e. blood, liver, skeletal muscle, using viral and electroporation based approaches (Andre and Mir 2004; Gissel 2010). Skeletal muscle is a particularly attractive host since the large muscle mass can be (ab)used for the local production of biological factors (Coelho-Castelo et al. 2006; Louz et al. 2005). It is generally accepted that methods should be developed to prevent the abuse of this technology in Sports (Gassmann et al. 2005; Haisma and de Hon 2006).

The WADA banns the use of gene doping based on the definition of 'the non-therapeutic use of genes, genetic ele- 
ments and/or cells that have the capacity to enhance athletic performance' (Unal and Ozer Unal 2004; http://www. wada-ama.org). The doping code specifies that any biological material may be collected for the purposes of Doping Control (http://www.wada-ama.org). In reality, detection of gene doping will only be feasible in samples which can be collected based on accepted codes of practice such as urine and blood (Hartgens 2008). A number of direct and indirect methods of detection have been proposed (Baoutina et al. 2010; Haisma and de Hon 2006; Mitchell et al. 2009). Polymerase chain reaction (PCR) is among the most sensitive technology that would allow detecting gene doping (Baoutina et al. 2010). This is because it amplifies deoxyribonucleic acids (DNA) sequences of the introduced genetic vector or encoded transcript after reverse transcription of RNA (Kubista et al. 2006). Specificity is directed by the selective binding of PCR oligonucleotides (primer) to the matching sequences in the sense and anti-sense strand of the DNA template which define the outer borders of the amplicon. The amplification reaction bears the important advantage that the amount of the resulting amplicon is proportional to the initial DNA amount in the sample and lends itself to a quantitative evaluation through the use of an amplification standard.

A number of biological processes offer for the genetic enhancement of human performance. This includes DNAbased strategies to promote muscle growth via the activation of anabolic signal pathways (Striegel and Simon 2007). This has been shown for a number of factors, i.e. AKT, Erythropoietin (Gassmann et al. 2005; Haisma and de Hon 2006). Focal adhesion kinase (FAK) is part of the hypertrophy pathway which integrates hormonal and mechanical cues in a number of cell types, into the activation of the protein synthetic pathway (Klossner et al. 2009; Torsoni et al. 2003). FAK-mediated signalling is regulated through the phosphorylation of tyrosine residue 397 (pY397) of FAK which reflects the initiation of the phosphotransfer activity of FAK and allows recruitment of downstream targets (Hanks et al. 2003). The former posttranslational modification occurs in response to mechanical loading and endocrine factors such as growth factors (Durieux et al. 2009; Rozengurt 1995; Shyy and Chien 1997). Elevated pY397 content with increased loading of skeletal muscle precedes the phosphorylation of p70S6Kinase (Klossner et al. 2009) which upregulates ribosomal activity in a number of cell systems (Volarevic and Thomas 2001). The implication of FAK-mediated activation of hypertrophy signalling is visualized in the increase of mean crosssectional area of muscle fibres 7 days after transfection of anti-gravitational muscle by gene electro transfer with an expression construct for FAK (Durieux et al. 2009).

While the detection of genetic modification has been shown for virus-mediated transfection of skeletal muscle with expression construct for the secreted hematopoietic factor erythropoietin (Beiter et al. 2011; Ni et al. 2011) it is unlikely that this transfection technology would be a method of choice. This is explained by various drawbacks including the induction of immune responses that reduce the success of repeated applications, the costs of production of GMO quality vectors and possibly fatal consequences due to the virulence of the approach (Raper et al. 2003). In consequence, the possibilities for detection of gene doping by other means of transfection such as gene electrotransfer and net effects of gene transfection on muscle structure and function are not explored.

We hypothesized that the introduction of a naked DNA vector in skeletal muscle is detectable in the serum of transfected animals as injected introduced plasmid may disseminates into the body (Coelho-Castelo et al. 2006). This was tested using established gene therapy for the amplification of load-dependent hypertrophy signalling via overexpression of an exogenous FAK homologue from gallus gallus in rat soleus muscle from a constitutively active expression plasmid (Durieux et al. 2009). Exogenous and endogenous FAK sequence was quantified with PCR on isolated DNA using species-specific primer pairs. Results were validated against spiking controls and plasmid standard and measures after early time points of transfection.

\section{Methods}

Study design

Soleus and gastrocnemius muscle of 3-month-old male Wistar rats was transfected with expression plasmid for chicken FAK by gene electrotransfer. Blood and muscle samples were collected and DNA was extracted for amplification with polymerase chain reaction using specific primers. In addition, muscle samples were processes for detection of FAK and muscle fibre size in cryosections and p70S6K phosphorylation in immunoblots. Samples from non-transfected animals served as controls. The timeline of muscle sampling is shown in Fig. 1a.

\section{Muscle transfection}

Soleus muscles of anaesthetized rats (3\% isoflurane) were transfected essentially as described (see Fig. 1b, c; Durieux et al. 2009). In brief, soleus muscle was surgically exposed and cytomegalovirus-driven expression construct for the chicken FAK homologue, pCMV-FAK $(1 \mathrm{mg} / \mathrm{ml})$ or an equivalent amount of empty pCMV-plasmid was injected into the belly portion of the muscle. Plasmid uptake in muscle fibres was stimulated by three trains of 80 pulses of $100 \mu$ s duration, each at $100 \mathrm{~mA}$, at two sites lateral to the 
Fig. 1 Methodology to transfect skeletal muscle. a Experimental protocol; b, c Images showing the plasmid injection (a) and electropulsing (b) of rat soleus muscle

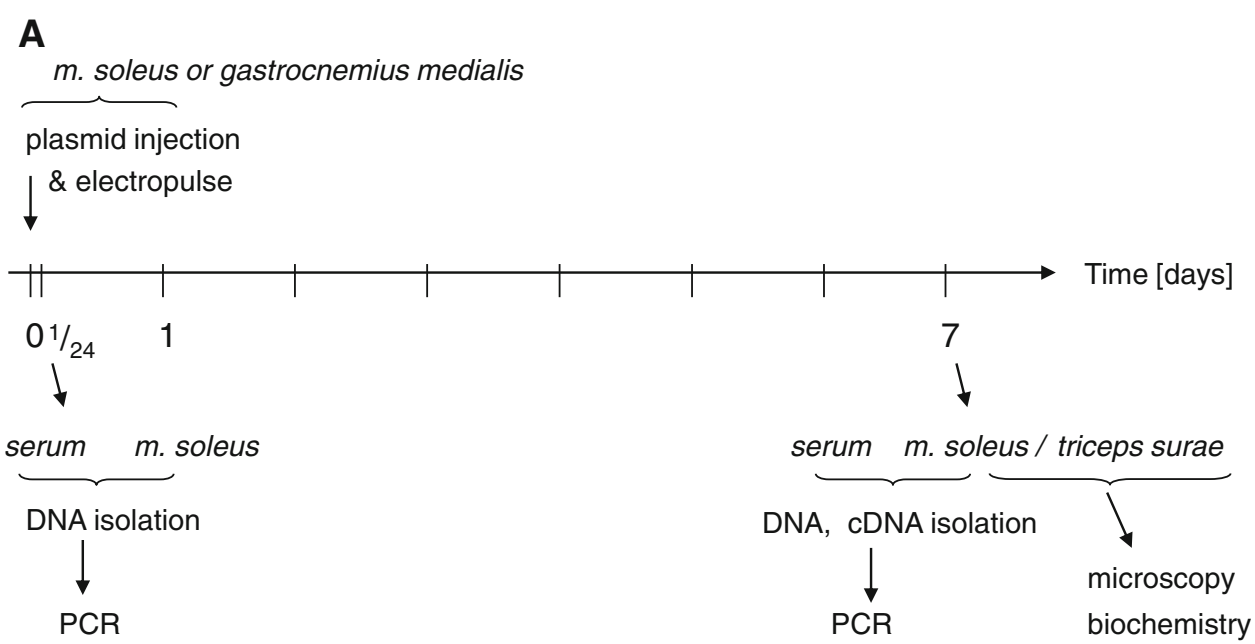

B

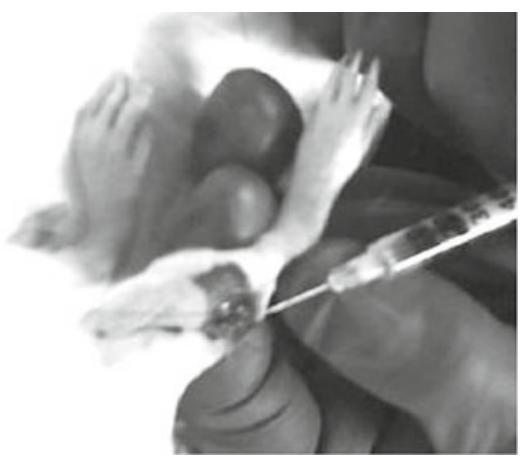

C

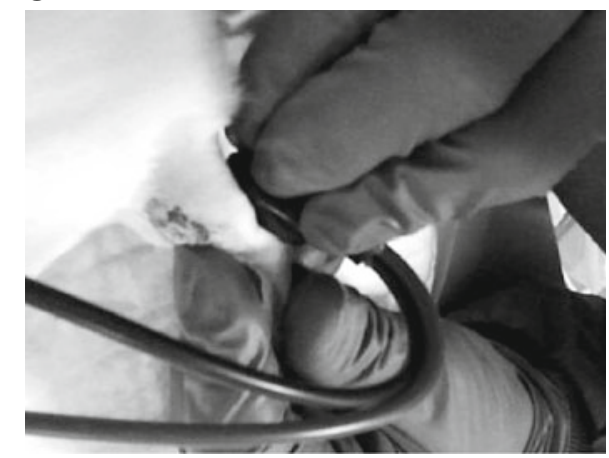

injection site using needle electrodes with the GET42 generator (EIP Electronique et Informatique du Pilat, Jonzieux, France). The skin and fascia were closed with sutures and the animals transferred to single cages. After a day of reduced cage activity, the behaviour and food intake of transfected rats could not be distinguished to non-transfected animals. Measures of body weight demonstrated that the animals continued to take on weight in the order of $0.3 \%$ per day in the week after gene transfer.

In addition, gene electrotransfer was carried out on gastrocnemius medialis muscle. $150 \mu \mathrm{g}$ plasmid in $150 \mu \mathrm{l}$ was injected in the mid portion of the muscle. Subsequently three trains of 100 pulses of $100 \mu$ s duration, each at $50 \mathrm{~mA}$, were delivered at two locations lateral to the injection site.

"Principles of laboratory animal care" (NIH publication No. 86-23, revised 1985) were followed. The experiments were performed at the Insel-Hospital of Berne, in Switzerland, under approval by the Animal Protection Commission of the Canton Bern, Switzerland and at the Medical School of the University of Birmingham with permission of the Home office. The animals were housed in pairs in cages in a care facility with a 12:12-h light/dark cycle at a constant temperature of $22^{\circ} \mathrm{C}$ and maintained on a diet of standard chow with water ad libitum.
Collection of muscle samples

Soleus and gastrocnemius muscles were extracted from anesthetized rats $1 \mathrm{~h}$ and 7 days after transfection. For removal of the soleus muscle, a circumferential incision was made to the skin below the calcaneus. Subsequently, the distal end of the soleus and its attached tendon were exposed with the help of forceps. Bleeding started at this point. The Achilles tendon part of the soleus was grabbed with a bent clamp and cut with a scalpel. The soleus muscle was successively lifted from its bed towards the heel of the knee with the help of the attached clamp. When the 'semitendinous' part became apparent at the proximal end of the soleus muscle, it was cut with a pair of scissors and the muscle was extracted. Gastrocnemius muscles were harvested in separate experiments where the triceps surae (composed of gastrocnemius medialis and lateralis, soleus and plantaris) was removed as a whole. This isolation was achieved by isolating the Achilles tendon as described above for soleus muscle but then the different heads of the muscle-tendons were bundled with a surgical thread before securing them with a clamp. Subsequently, the entire triceps surae was lifted from its bed with the help of a scalpel, trimming off residuals of attached soft tissue. When the knee heel was apparent, the muscle heads were capped and 
the triceps removed. Excised muscles were frozen in liquid nitrogen cooled isopentane and stored at $-80^{\circ} \mathrm{C}$ until use.

Sampling of blood serum

Blood $(0.2 \mathrm{ml})$ was drawn with a syringe from the tissue bed' where the removed, transfected soleus muscle used to reside before the surgery. Another $0.4 \mathrm{ml}$ of blood was drawn from the left ventricle, of euthanized animals and rapidly transposed in heparinized tubes, frozen in liquid nitrogen, and stored at $-20^{\circ} \mathrm{C}$ until analysis.

\section{DNA extraction}

Deoxyribonucleic acids were extracted with the DNeasy Blood and Tissue Kit (QIAGEN Ltd. QIAGEN House Fleming Way Crawley West Sussex, RH10 9NQ, UK). Blood samples were extracted using the spin-column non-nucleated erythrocytes protocol described in the kit handbook and available at DNeasy website (DNeasy 2006, http://www.qiagen.com). Final sample volume was $100 \mu$ l. On average, $0.02 \mu \mathrm{g}$ total DNA was isolated per $0.2 \mathrm{ml}$ of serum. Soleus muscle samples were sectioned in a cryostat (Leica) at a thickness of $12 \mu \mathrm{m}$ to aid the lysation process. An estimated volume of $25 \mathrm{~mm}^{3}$ of sample was used during the protocol for the extraction of DNA from animal tissues. Extracted DNA was stored in $-20^{\circ} \mathrm{C}$ freezer.

\section{Polymerase chain reaction}

Reverse and forward oligonucleotide primers were designed using Primer Express software (PE Biosystems, Rotkreuz, Switzerland) and Basic Local Alignment Search Tool (BLAST; http://blast.ncbi.nlm.nih.gov/Blast.cgi) to detect unique sequences in the homologous sequence of the respective rat (Genbank AF020777) of chicken FAK homologues (Genbank M86656). The sequence of the two primer pairs is shown in Fig. 2a. Due to the imposed limitations, a chance for primer dimers existed.

PCRs were run on the isolated DNA with FAK-specific primers with SYBR Green Master Mix chemistry (Bio-Rad Laboratories Ltd, Hemel Hempstead, UK) on a fluorescence PCR machine (DNA machine, Bio-RioRad) operated by Opticon 3 monitor software. Reactions were run with $1 \mu \mathrm{l}$ of DNA sample in a final volume of $21 \mu \mathrm{l}$ within 96well plates based on the Opticon 3 monitor instructions. The PCR protocol comprised an activation step $\left(95^{\circ} \mathrm{C}\right.$ for $5 \mathrm{~min})$ and 50 cycles of denaturing $\left(95^{\circ} \mathrm{C}\right.$ for $\left.15 \mathrm{~s}\right)$, annealing $\left(55^{\circ} \mathrm{C}\right.$ for $\left.1 \mathrm{~min}\right)$ followed by a read of the fluorescence on the well. This was terminated by melting curve analysis with a protocol rising the temperature in steps of $0.5^{\circ} \mathrm{C}$ (held $1 \mathrm{~s}$ ) from 55 to $95^{\circ} \mathrm{C}$. The cycle of threshold detection $C_{\mathrm{t}}$ was estimated using the Opticon 3 software.
The efficiency of the PCR amplification was quantified in titration experiments with pCMV-FAK plasmid and chicken FAK-specific primers. The efficiency was assessed from the slope of linear relationship between the input DNA (i.e. plasmid) and the $C_{\mathrm{t}}$ of PCR amplification. Plasmid DNA amounts were determined against a standard curve as determined with PCR on a dilution series of pCMV-FAK plasmid. For the spiking experiments, pCMVFAK plasmid was mixed with DNA sample from blood serum of a non-transfected, control rat at a concentration of $50 \mathrm{femtogram} /$ microliter.

Immunoblotting

Soleus muscles were cryosectioned at $12 \mu \mathrm{m}$ and total homogenate was prepared in modified RIPA buffer ( $1 \%$ NP-40, $0.25 \%$ deoxycholate, $50 \mathrm{mM}$ Tris $\mathrm{HCl}, \mathrm{pH} 7.4$, $1 \mathrm{mM}$ EDTA, $150 \mathrm{mM} \mathrm{NaCl}, 1 \mathrm{mM} \mathrm{NaF}, 1 \mathrm{mM}$ PMSF, $1 \mathrm{mM}$ sodium orthovanadate, $1 \mu \mathrm{g} / \mathrm{ml}$ leupeptin, $2 \mu \mathrm{g} / \mathrm{ml}$ pepstatin, $1 \mu \mathrm{g} / \mathrm{ml}$ aprotinin; all reagents were received from Sigma-Aldrich (Dorset, England). Protein concentration was quantified with Bicinchoninic acid assay reagents against BSA standard (Pierce, Thermo Fisher Scientific, Cramlington, UK). Twenty micrograms of protein were separated by SDS-PAGE, western blotted onto protran nitrocellulose (Schleicher \& Schuell BioScience GmbH, Whatman Group Hahnestrasse 3, D-37586 Dassel, Germany) and subjected to immunodetection with p70S6K antibody (C-18, Santa Cruz, CA, USA) as described (Klossner et al. 2009). Signal detection was carried out with enhanced chemoluminescence using a Geldoc system that was operated using Quantity One 1-D analysis software 4.6.1 (Bio-Rad Laboratories Ltd, Hemel Hempstead, UK). Signal intensity of the p70S6K bands was determined using the rectangle density mode and background from an empty sample lane was subtracted.

Immunofluorescence

Detection of FAK in soleus and gastrocnemius muscle was carried out essentially as described in Klossner et al. (2009). In brief, $12-\mu \mathrm{m}$ cryosection were incubated with the FAK N-terminal antibody A-17 from rabbit (1:100 dilution, Santa Cruz) and Alexa Fluor 488-coupled secondary anti-rabbit antibody (DAKO, Denmark). Fluorescence and digital phase contrasts were recorded using a TCS SP5 confocal microscope (Leica Microsystem CMS, Mylton Keynes, UK). Mean cross-sectional area of fibres overexpressing FAK or showing background levels in stained cryosections (=not-overexpressing fibres) was quantified using the macro application of Leica Application Suite (LAS). Towards this end, random images were taken at a 20 -fold magnification from microscopic fields 
A

cFAK taatgatgttattggtcggattgagaacggtgagcggctcccatgcctccgaactgccetccaccct

attactacaataaccagcctactcttgccactcgccgaggggtacggaggcttgacgggagggtggga

rFAK tgaccgatccaatgacaaggtatatgaaatgtgacgggcctagtgaaggctgtcattgagatgtccagcaaaatccaaccagctcctce actggctaggttactgttccatatacttttacactgccogatcacttccgacagtaactctacaggtcgttttaggttggtcgaggagg

B

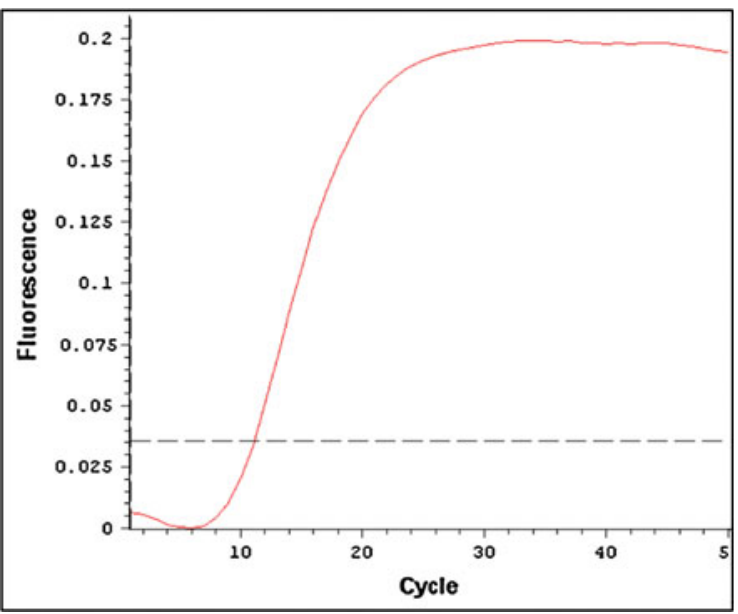

D

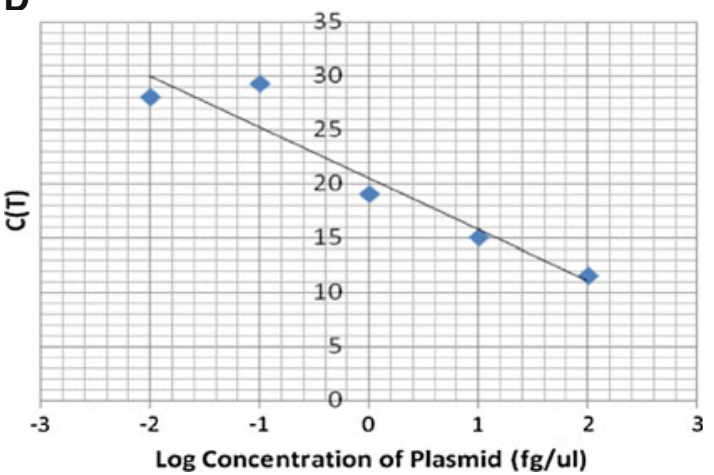

Fig. 2 a Sequence alignment of the primers used to amplify the nucleotide sequence of the exogenous (chicken) FAK and endogenous (rat) FAK. b, c melting and amplification curve of 100 femtogram/microliter pCMV-FAK plasmid as amplified with chicken specific FAK-prim-

of the part in the cross section of pCMV-FAK transfected muscle showing FAK overexpression. FAK-transfected fibres were identified in each image. Then the circumference-as identified with the digital phase contrasts-was circled with the quantification tool of LAS to estimate cross-sectional area. For each image, the cross section of clearly demarcated FAK-overexpressing and not-overexpressing muscle fibres was measured. Raw data were exported for statistical analysis.

\section{Statistics}

$T$ tests were carried out to compare the calculated plasmid amounts between the samples. The significance was set to $5 \%$. Results are graphical represented.

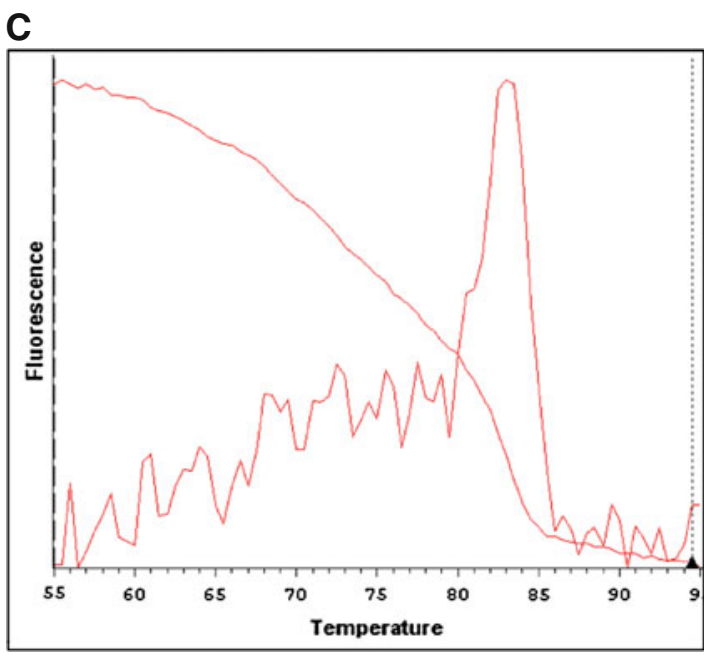

er. d Titration curve indicating the linear amplification of the pCMVFAK amplicon with chicken specific FAK primer between 0.01 and 100 femtogram plasmid per microliter

\section{Results}

Muscle transfection

Figure 1 visualises the methodology employed to transfect soleus muscle of rats with cytomegalovirus-driven expression construct for the chicken FAK homologue, pCMVFAK. One hundred and fifty micrograms of plasmid were injected into the exposed muscle which uptake in muscle fibres was subsequently stimulated by electric pulses (electro gene transfer). The biological effect of transfection was analyzed 7 days later. Microscopic examination identified muscle fibres which demonstrated sizeably increased expression of FAK protein in the sarcoplasm (Fig. 3a, c). M. soleus being injected with empty, pCMV-plasmid did 
Fig. 3 Biological effect of muscle transfection with expression construct for the chicken FAK homologue. Microscopic examination of fluorescently stained FAK (light grey/red) in soleus (a) and gastrocnemius medialis muscle (b) 7 days after gene electrotransfer with pCMV-

FAK plasmid. Mean cross-sectional area of FAK overexpressing ('+') and not-overexpressing muscle fibres ('-') of $m$. soleus (c, $n=33$ vs. 94 fibres) and $m$. gastrocnemius (d, $n=21$ vs. 44 fibres). The data were collected from cross sections of transfected muscle from three animals, each. e Example of p70S6K in $20 \mu \mathrm{g}$ total protein from empty pCMV ('-') and pCMV-FAK ('+') transfected soleus muscle sample as detected by immunoblotting. f Fold-change in p70S6K protein with pCMV FAK transfection of soleus muscle. Asterisk denotes $p<0.05$ versus the non-transfected muscle (fibre)
A
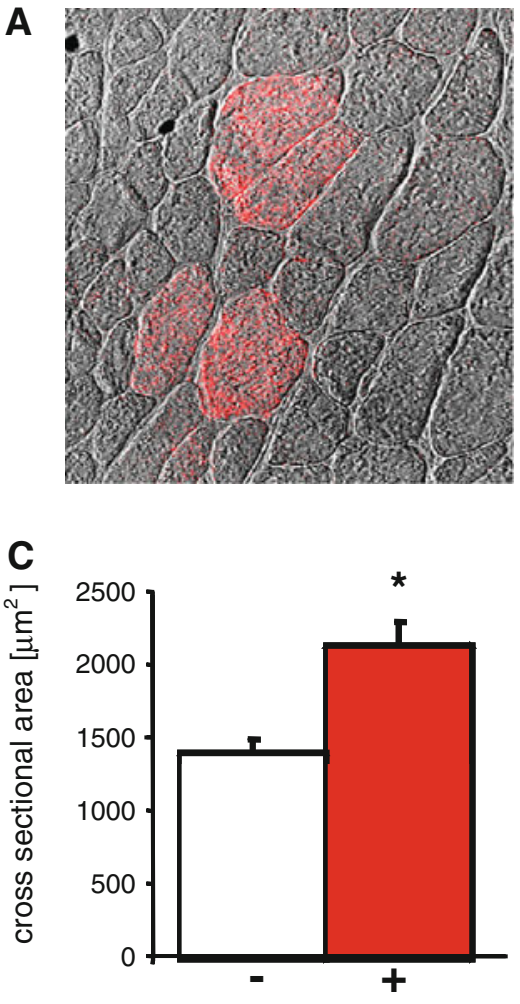

FAK overexpression
B

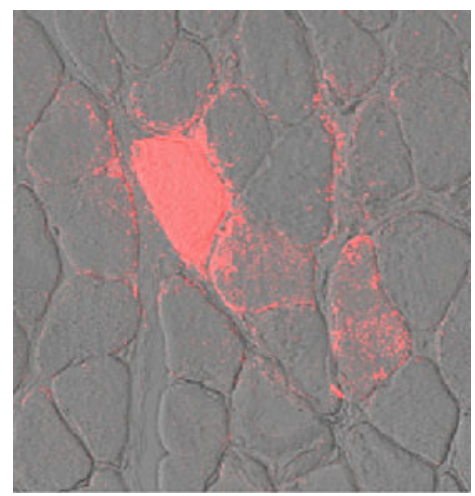

D

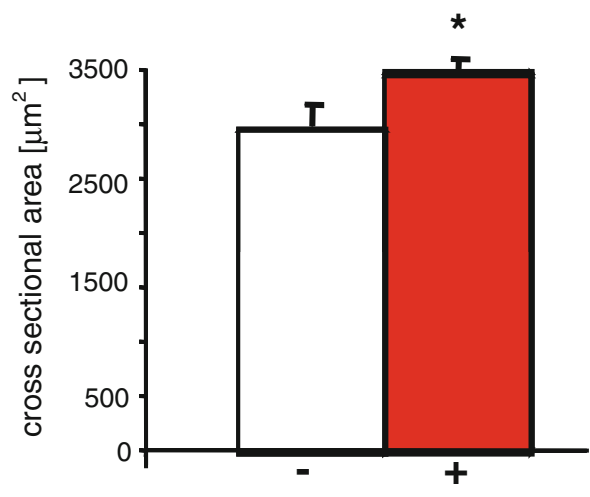

FAK overexpression

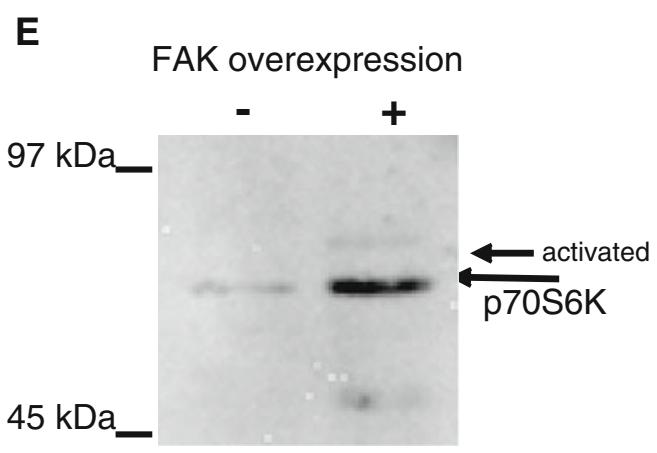

not demonstrate fibres with elevated FAK signal (data not shown). Quantification revealed a transfection efficiency of $12 \%$ in the targeted soleus muscle. The targeted muscle fibres demonstrated on average a 5.5-fold increase in FAK signal intensity (i.e. expression). Their mean cross-sectional area was $52 \%$ larger compared to fibres with baseline FAK levels. Concomitantly, with pCMV-FAK transfection, the protein levels of p70S6K were 2.1-fold increased (Fig. 3e, f).

We transfected the medial portion of gastrocnemius muscle with pCMV-FAK (Fig. 3b). Transfection efficiency was $4.5 \%$ in the targeted muscle portion. The fluorescent signal for FAK was 73-fold elevated in transfected fibres.
Mean cross-sectional area was $16 \%$ larger in muscle fibres which overexpressed FAK (Fig. 3d).

Establishing the detection of expression construct

An oligonucleotide pair was designed to detect the expression plasmid pCMV-FAK with PCR-based amplification of a unique nucleotide segment in the exogenous (chicken FAK) DNA sequence of the plasmid (Fig. 2a). The criteria for the nucleotide pair were that the primers complied with the default settings for SYBR and Taqman biochemistry and would allow separating the exogenic from the endogenous (rat) FAK homologue. 
Fig. 4 PCR detection of exogenous FAK in DNA samples from rats which soleus muscle was transfected with pCMV-FAK. a, b Screen plots of amplification and melting curve for a PCR using chicken FAK primer pairs on serum DNA from a nontransfected rat muscle being spiked with 50 femtogram/ microliter pCMV-FAK. c, d Amplification and melting curves of a PCR of a cDNA sample from pCMV-FAK-transfected rat soleus muscle. e, f PCR using rat FAK-specific primers on serum DNA of a rat with pCMV-FAK transfected soleus muscle (dark/red line) and serum DNA of a non-transfected control rat. Note the similar threshold of detection for the endogenous FAK gene transcript
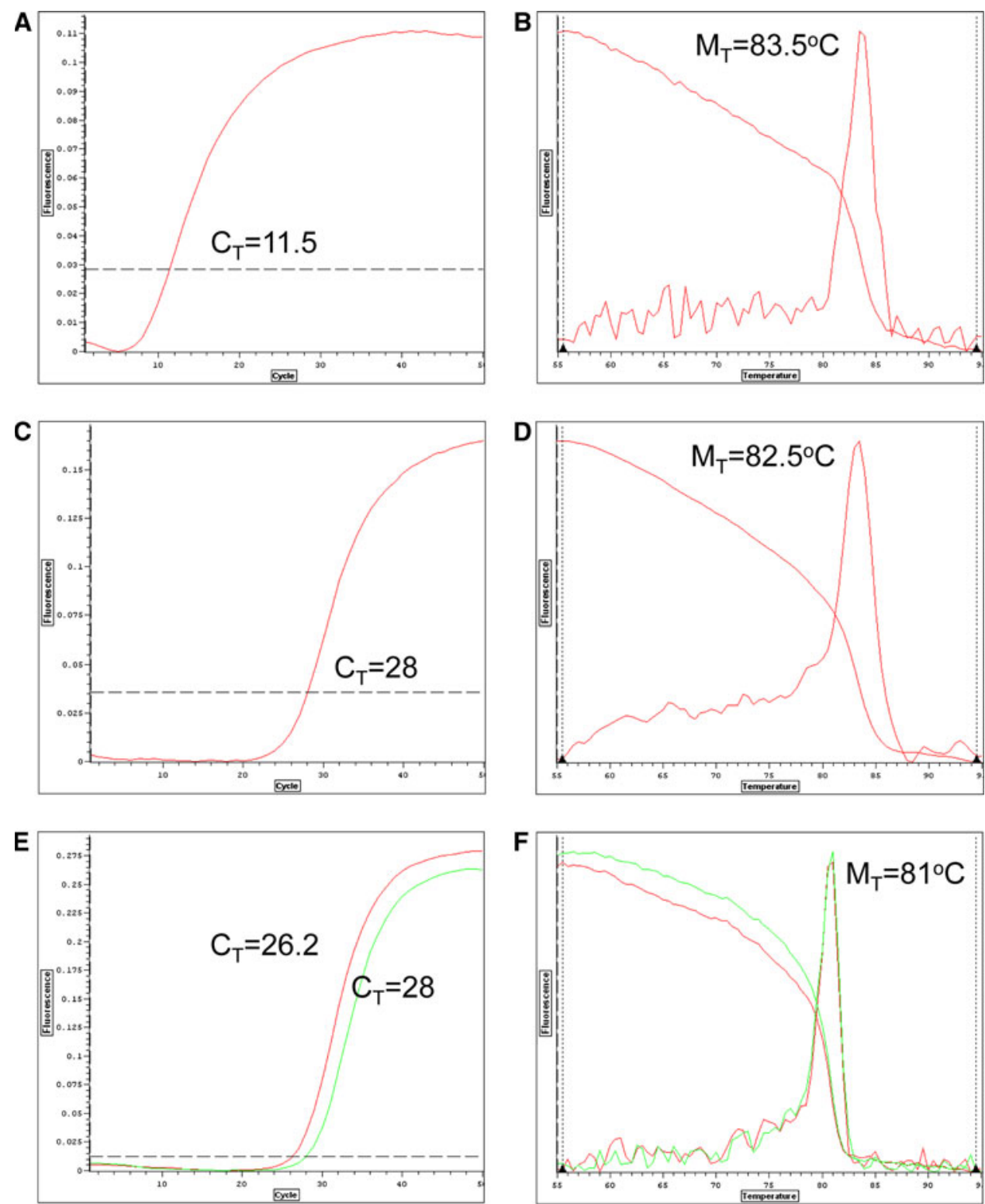

These setup experiments demonstrated the linear amplification of a specific amplicon with a length of $69 \mathrm{bp}$ and a melting temperature of $83.5^{\circ} \mathrm{C}$ in PCR with pCMV-FAK plasmid DNA (Fig. 2b). The calculated threshold of detection was estimated to situate at $0.01 \mathrm{femtogram} /$ microliter.

Quantifying plasmid levels in blood of transfected rats

Spiking experiments with DNA from blood serum (='serum DNA') from non-transfected rats showed that the detection of the pCMV-FAK plasmid is feasible (Fig. 4a). In serum DNA samples from non-transfected rats detection, endogenous background was amplified in late cycle of the PCR $\left(C_{\mathrm{t}}=38\right.$; data not shown $)$.

We analyzed DNA from blood serum of somatically transfected rats to assess whether the inserted plasmid is detectable with PCR using the chicken FAK-specific primers. The plasmid could be detected in serum DNA that had been collected from the muscle bed $1 \mathrm{~h}$ after injection of $75 \mu \mathrm{g}$ pCMV-FAK into soleus muscle and surgery (the 'muscle site'; Fig. 5a). The amplicon was not detected above the background in DNA from blood collected from heart after the same time point of transfection.

In serum DNA that had been collected 7 days after electro gene transfer of soleus muscle, the chicken FAK amplicon could not be detected above background levels seen in non-transfected rats (Fig. 5b).

A second oligonucleotide primer pair was designed to detect a segment in the endogenous (rat) FAK transcript (Fig. 2a). Control experiments with this probe showed that endogenous FAK was detectable in serum DNA independent of muscle transfection (Fig. 4e, f). 


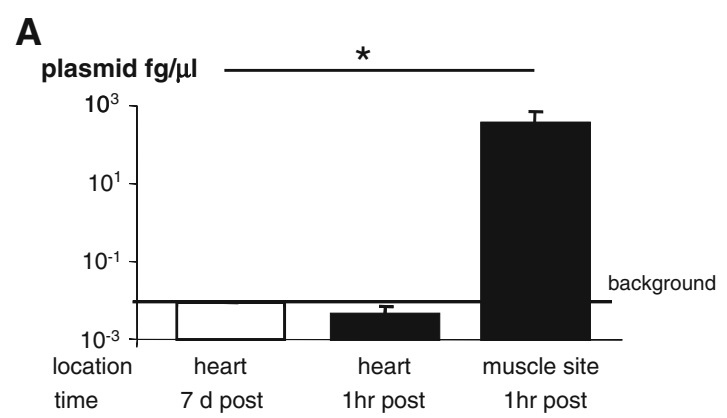

Fig. 5 Time course for PCR detection of exogenous FAK in serum of transfected rats. Calculated amount of pCMV-FAK plasmid in blood serum (a) and muscle samples (b) from soleus muscles of transfected and non-transfected rats. Serum samples were collected close to the

Quantifying plasmid in targeted muscle

PCR experiments with transfected muscle sample showed that the detection of pCMV-FAK plasmid is feasible in DNA being isolated from soleus muscle $1 \mathrm{~h}$ after plasmid injection. Seven days after transfection, detection of the chicken FAK amplicon was retained in cDNA of the soleus muscle being transfected with pCMV-FAK (Figs. 4c, 5b).

\section{Discussion}

Doping represents a major threat for health and interferes with the ideal of fairness in athletic competition. While it is not currently understood to which extent gene doping is currently in use and possibly fictive (Andersen et al. 2000; Glogger 2008), gene doping is on the list of banned interventions without firm methods for its detectability being established (Gassmann et al. 2005). Towards this end, we addressed in a test system for hypertrophy signalling via focal adhesion kinase, whether more recently introduced blood tests would be an option to detect somatic gene therapy with sensitive PCR technology. Our experiments provide novel evidence for anabolic reactions in FAK transfected muscle fibres and emphasize that detection of exogenous gene sequences is possible on pure DNA that has been isolated with commercial kits from the hypertrophied muscle after transfection. By contrast, detection is not necessarily feasible in DNA being prepared from blood serum. These observations have a number of implications for the biological effects and diagnostics of gene doping in Sports.

Biochemical and cellular measures of the targeted soleus muscle imply that the enhancement of FAK expression by genetic means elevates cross-sectional area of muscle fibres and increases in the expression of the phosphotransfer enzyme p70S6kinase (Fig. 3e, f). This signalling factor cor-

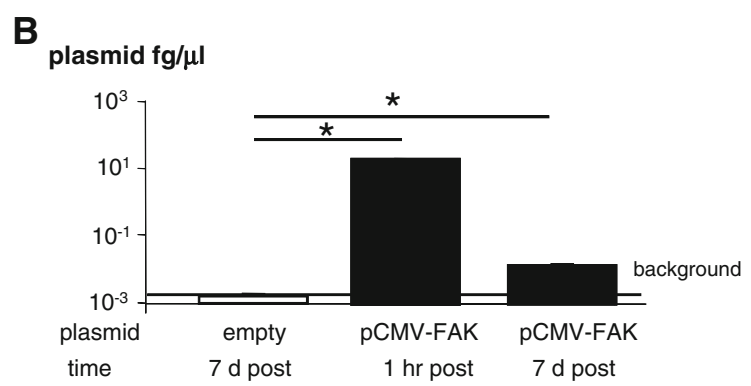

site of pCMV-FAK plasmid injection ('muscle site') or the left ventricle ('heart') 1 day or 7 days after plasmid injection. Asterisk indicates $p<0.05$ based on a $T$ test

relates strongly with the degree of muscle hypertrophy (Zanchi and Lancha 2008). We have previously shown that reloading of atrophied soleus muscle increases phosphorylation of p70S6K at regulatory amino acids (Klossner et al. 2009). This finding indicates that the anabolic effect of FAK overexpression in the postural muscle under study reflects the amplification of load-induced hypertrophy signalling.

We also targeted gastrocnemius medialis muscle and found that the cross-sectional area of FAK-transfected muscle fibres was significantly increased compared to non-transfected muscle fibres as well $(+16 \%, p=0.04)$. This degree of hypertrophy is smaller than that seen for transfected soleus (52\%) muscle despite heavier elevations in FAK expression levels (73- vs. 7-fold). This discrepancy possibly reflects different fibre type composition in soleus and gastrocnemius medialis muscle (DeRuiter et al. 1996; Fluck et al. 2002). Using an antibody that is now understood to detect the activated FAK molecule (Durieux et al. 2009), we have shown that FAK activation relates to the recruitment of muscle fibres types (Fluck et al. 2002). The predominately slow type motor units in soleus muscle are frequently implicated in load-bearing muscle action with daily movement patterns (i.e. 22-35\% of the total time (Hennig and Lomo 1985). By contrast, the fast type motor units of gastrocnemius muscle are mainly involved in propulsion and thus far less frequently activated in laboratory animals (i.e. $<5 \%$ of total activity). The comparison of the two muscle types supports the notion that the outcome of recruitment-induced FAK signalling is different in slow and fast type muscle fibres of anti-gravitational muscle.

Having established the principal feasibility of detecting introduced DNA in serum via amplification of exogenous DNA segment, we were unable to specifically amplify the same sequence in serum collected 7 days after the intervention. Similarly, the pCMV-FAK plasmid was not detectable in serum DNA from samples collected from the 
left ventricle $30 \mathrm{~min}$ after plasmid injection in soleus muscle, but when the exogenous FAK sequence was detectable is serum collected close to the site of DNA injection (Fig. 5a). Meanwhile, the sequence of the endogenous FAK could be amplified at a similar level in serum DNA of non-transfected control rat as in serum from rats with pCMV-FAK transfected soleus muscle (Fig. 4e, f). The positive control experiment indicates that the DNA yield and quality were not limiting factors in our experiments. Our data compare to observations commenting on the narrow time window under which injected DNA can be detected in the circulation (Baumgartner et al. 2009). There was minor surgical injury to the transfected soleus muscle. We, therefore, estimate that the pCMV-FAK plasmid that could be detected in serum sampled close to the site of DNA injection originates from the remainder of DNA material which leaked from the electropulsed soleus into the tissue bed or blood normally entering or exiting the soleus muscle. Our observations indicate that dilution effects in the serum compartment or eventually rapid DNA elimination reduce the chances of detecting gene doping in blood samples collected distant to the site of local genetic treatment.

This relates to a very recent report demonstrating that intramuscularly injected conventional plasmid is rapidly eliminated (Ni et al. 2011). Equally we noted that the signal for exogenous FAK was importantly reduced in transfected soleus muscle 7 days after transfection. The findings imply that the detection of somatic gene therapy with the employed PCR technology is confined to a narrow spatialtemporal window after the intervention.

Dilution experiments with pCMV-FAK plasmid showed that the exogenous FAK sequence is not detectable with our PCR setup above background below 0.01 femtogram/microliter reaction mix. PCR experiments with water only imply that the background is explained by the amplification of primer dimers (data not shown). Due to the imposed restrictions for amplicon length, the background PCR product was indistinguishable based on melting temperature to the specific product from pCMV-FAK plasmid amplification. We calculate that the threshold level for detection of exogenous FAK by PCR corresponds to a ratio of plasmid to total DNA of $1: 10,000$ (w/w). Quantitative considerations further indicate that only a minor portion of the original $150 \mu \mathrm{g}$ of the plasmid being injected in soleus muscle (i.e. 1/125,000) is detectable in blood serum. Based on the volumes used to inject plasmid before electropulsing, we estimate that the detection of injected plasmid would not be technically feasible below a concentration an injected amount of $50 \mu \mathrm{g}$ in our rat system. Thus when larger targeted tissues are targeted with similar DNA amounts, it is unlikely detectable with the employed PCR technology.

\section{Conclusions}

Our assays in a model system for somatic transgenesis indicate that the option to detect gene doping with conventional PCR is gone 7 days after the DNA intervention. Given this short window of detection, this indicates that a change in current sampling practice is indicated to provide anti-doping panels with the diagnostic power to detect gene doping. This should involve the collection of muscle biopsies within the first week after DNA injection at the suspected site of the genetic intervention.

Acknowledgments The assistance of Dr. Stuart Egginton (University of Birmingham) for the animal experiments and Msc Wouter Eilers for help in initial PCR experiments is greatly acknowledged.

\section{References}

Andersen JL, Schjerling P, Saltin B (2000) Muscle, genes and athletic performance. Sci Am 283:48-55

Andre F, Mir LM (2004) DNA electrotransfer: its principles and an updated review of its therapeutic applications. Gene Ther 11 (Suppl 1):S33-S42

Baoutina A, Coldham T, Bains GS, Emslie KR (2010) Gene doping detection: evaluation of approach for direct detection of gene transfer using erythropoietin as a model system. Gene Ther 17:1022-1032

Baumgartner I, Chronos N, Comerota A, Henry T, Pasquet JP, Finiels F, Caron A, Dedieu JF, Pilsudski R, Delaère P (2009) Local gene transfer and expression following intramuscular administration of FGF-1 plasmid DNA in patients with critical limb ischemia. Mol Ther 17(5):914-921

Beiter T, Zimmermann M, Fragasso A, Hudemann J, Niess AM, Bitzer M, Lauer UM, Simon P (2011) Direct and long-term detection of gene doping in conventional blood samples. Gene Ther 18:225-231

Coelho-Castelo AA, Trombone AP, Rosada RS, Santos RR Jr, Bonato VL, Sartori A, Silva CL (2006) Tissue distribution of a plasmid DNA encoding Hsp65 gene is dependent on the dose administered through intramuscular delivery. Genet Vaccines Ther 4:1

DeRuiter CJ, De Haan A, Sargeant AJ (1996) Fast-twitch muscle unit properties in different rat medial gastrocnemius muscle compartments. J Neurophysiol 75(6):2243-2254

Durieux AC, D'Antona G, Desplanches D, Freyssenet D, Klossner S, Bottinelli R, Fluck M (2009) Focal adhesion kinase is a loaddependent governor of the slow contractile and oxidative muscle phenotype. J Physiol 587:3703-3717

Filipp F (2007) Is science killing sport? Gene therapy and its possible abuse in doping. EMBO Rep 8:433-435

Fluck M, Ziemiecki A, Billeter R, Muntener M (2002) Fibre-type specific concentration of focal adhesion kinase at the sarcolemma: influence of fibre innervation and regeneration. J Exp Biol 205:2337-2348

Gassmann M, Rusconi S, Flueck M (2005) Observatorium Gene doping. Bundesamt für Sport (BASPO), Switzerland

Gissel H (2010) Effects of varying pulse parameters on ion homeostasis, cellular integrity, and force following electroporation of rat muscle in vivo. Am J Physiol Regul Integr Comp Physiol 298:R918-R929

Glogger B (2008) Lauf um mein Leben. Rowohlt Taschenbuch. Verlag, Reinbeck bei Hamburg 
Haisma HJ, de Hon O (2006) Gene doping. Int J Sports Med 27:257266

Hanks SK, Ryzhova L, Shin NY, Brabek J (2003) Focal adhesion kinase signaling activities and their implications in the control of cell survival and motility. Front Biosci 8:d982-d996

Hartgens F (2008) Medication, athletes and doping regulations. Ned Tijdschr Geneeskd 152:1844-1848

Hennig R, Lomo T (1985) Firing patterns of motor units in normal rats. Nature 314:164-166

Klossner S, Durieux AC, Freyssenet D, Flueck M (2009) Mechanotransduction to muscle protein synthesis is modulated by FAK. Eur J Appl Physiol 106:389-398

Kubista M, Andrade JM, Bengtsson M, Forootan A, Jonak J, Lind K, Sindelka R, Sjoback R, Sjogreen B, Strombom L, Stahlberg A, Zoric N (2006) The real-time polymerase chain reaction. Mol Aspects Med 27:95-125

Louz D, Bergmans HE, Loos BP, Hoeben RC (2005) Cross-species transfer of viruses: implications for the use of viral vectors in biomedical research, gene therapy and as live-virus vaccines. J Gene Med 7:1263-1274

Mitchell CJ, Nelson AE, Cowley MJ, Kaplan W, Stone G, Sutton SK, Lau A, Lee CM, Ho KK (2009) Detection of growth hormone doping by gene expression profiling of peripheral blood. J Clin Endocrinol Metab 94:4703-4709

Ni W, Le Guiner C, Gernoux G, Penaud-Budloo M, Moullier P, Snyder RO (2011) Longevity of rAAV vector and plasmid DNA in blood after intramuscular injection in nonhuman primates: implications for gene doping. Gene Ther

Raper SE, Chirmule N, Lee FS, Wivel NA, Bagg A, Gao GP, Wilson JM, Batshaw ML (2003) Fatal systemic inflammatory response syndrome in a ornithine transcarbamylase deficient patient following adenoviral gene transfer. Mol Genet Metab 80:148-158

Rozengurt E (1995) Convergent signalling in the action of integrins, neuropeptides, growth factors and oncogenes. Cancer Surv 24:81-96

Shyy JY, Chien S (1997) Role of integrins in cellular responses to mechanical stress and adhesion. Curr Opin Cell Biol 9:707-713

Striegel H, Simon P (2007) Doping. High-tech cheating in sport. Internist (Berl) 48:737-742

Sweeney HL (2004) Gene doping. Sci Am 291:62-69

Torsoni AS, Constancio SS, Nadruz W Jr, Hanks SK, Franchini KG (2003) Focal adhesion kinase is activated and mediates the early hypertrophic response to stretch in cardiac myocytes. Circ Res 93:140-147

Unal M, Ozer Unal D (2004) Gene doping in sports. Sports Med 34:357-362

Volarevic S, Thomas G (2001) Role of S6 phosphorylation and S6 kinase in cell growth. Prog Nucleic Acid Res Mol Biol 65:101127

Zanchi NE, Lancha AH Jr (2008) Mechanical stimuli of skeletal muscle: implications on $\mathrm{mTOR} / \mathrm{p} 70 \mathrm{~s} 6 \mathrm{k}$ and protein synthesis. Eur $\mathbf{J}$ Appl Physiol 102:253-263 